\title{
Reducing the Road Side Accidents and Collisions using the Connected Vehicle Technology
}

\author{
Bitra Renuka $^{*}$, B Gayathri Devi², G Gopika ${ }^{3}$ \\ $1^{*}$ Assistant Professor ,ECE Dept, Vignan's Lara Institute of Technology \& Science, Vadlamudi, Guntur, India. \\ ${ }^{2,3}$ M.Tech Student, ECE Dept, Vignan's Lara Institute of Technology \& Science, Vadlamudi, Guntur, India. \\ *Corresponding Author: Bitra Renuka, Assistant Professor ,ECE Dept, Vignan's Lara Institute of \\ Technology \& Science, Vadlamudi, Guntur, India.
}

\begin{abstract}
Road hazards take place every day in big cities. Sometimes, these accidents are fatal. Two major factors causing road accidents include high traffic in roads and rash driving. Generally, this is a cyclist and motor cyclist who are victims of the dangers. Sometimes, pedestrians are also killed in these accidents. Driver error is often the result of "distraction driving". The most common cause of driver distraction is the use of cell phones. According to the National Safety Council (NSC), 25\% of all automobile crashes are related to cell phone use, by phone or texting.

Traffic on highways has increased so much; it is often difficult to cross a highway. At the maximum of the day, we find a permanent flow of buses, trucks, cars, tempos, scooters, motor wheels and wheels. Vehicle drivers observe traffic jams. They try to overtake one another. Drivers who neglect buses and tracks are in the habit of drinking. Under the influence of alcohol they indulge in driving hives.they drive their vehicles at high speed. They notice speed limits. They are causing a road accident. Sometimes they are victims of such risks. Based on these conditions, road accidents can be reduced by using connected vehicle technology.
\end{abstract}

Keywords: Connected Vehicle Technology (CVT), Road accidents.

\section{INTRODUCTION}

In recent year connected vehicle technology (CVT) has been considered as the next big innovation platform for ITS .The connected vehicle technology means wireless communication between the motor vehicles.

In the proposed system RF transmitter and RF receiver are used for wireless communication between two vehicles. The secondary vehicle RF transmitter transmits the signals to the primary vehicle of the RF receiver. whenever it receives the signals from the RF transmitter the primary vehicle automatically stops by selecting operation on the mode switch, here four applications are needed for the operation i.e., blind spots, crash alerts, emergency vehicle and weather monitoring. Whenever an option is selected, that particular objective gets activated and it is useful for reducing the rate of accidents occurring in our day to day life.

Statistics show that the leading cause of death by injury is road traffic accidents. A survey report by World Health Organization highlights that every year more than 50,000 people in India are died due to road traffic accidents [3]. There are number of causes for which an accident can occur, some of them are lack of training institutes, use of mobile phone while driving, unskilled drivers, driving while intoxicated, bad road condition, overloading, and poor traffic management [4].

As the name implies it is a collision between an animal and a vehicle. Statistics shows that mortality of animals is increasing in North-East China due to accidents as compared to hunting [5]. Statistics shows that $67 \%$ drivers at Northern Tanzania felt that animal vehicle accidents were due to over speeding and night-time low visibility [6].

Hitting a pedestrian while driving a car is very common. A study highlighted that Islamabad Police Department reported $53.3 \%$ fatalities for pedestrian cases that are $56 \%$ of reported fatal traffic accidents [7]. The basic reasons of car accidents are over-speeding, casual behavior, reckless and unsafe driving [8].Due to slow movement of pedal cyclists, they are not seen by vehicles which over 
takes another car and suddenly hit the pedal cyclists. As motor cycle moves much faster than cycle so motor cyclists are less vulnerable than pedal cyclists [9].

\section{EXISTING SYSTEM}

In this existing system connected vehicle technology is introduced only on the real time traffic state estimation.

Connected vehicles (CVs) will reliably share the traffic condition data with surrounding vehicles through vehicle-to-vehicle $(\mathrm{V} 2 \mathrm{~V})$ communication and with transportation infrastructures through the vehicle-to- infrastructure (V2I) communication. Once the connected vehicle on-board units collect traffic data (e.g. vehicle positions, headway distances from leading vehicle front bumper to following vehicle front bumper etc.)[1][2] In an autonomous way at predetermined intervals, they transmit the data to roadside units (RSUs). Later, these data are further processed to traffic management center; RSUs are controlled by the TMC, where TMC assigns the parameters to control the information flow from the RSUs. Finally the information is forwarded to the other centers (i.e. maintenance and construction center, transportation information center, and emergency management center) to take necessary actions.

\section{Proposed System}

The main aim of this paper is to provide connected vehicles technology (cvt) and the extension is to reduce accidents by indicating crash alerts, avoid blind spots and also allow the emergency vehicles to pass. In the proposed system, two vehicles are needed for the operation. For the primary vehicle the modules such as IR sensor, buzzer, flash light, RF receiver and the display are interfaced to the raspberry pi development board. The secondary vehicle implemented by using arduino board. The modules interfaced to the secondary vehicle are LCD, buzzer, RF transmitter and rain sensor.

\subsection{Working of CVT}

The proposed system mainly contain two vehicles operate by IR sensor, RF transmitter and receiver.IR sensor and RF receiver connected to Raspberry pi board through GPIO (general purpose input) pins as primary vehicle. An SD (secure digital) is inserted into raspberry pi board card slot. The SD card having raspbian Jessie the source code files according to application. RF transmitter connected to ardunio board through GPIO as secondary vehicle. Power on the two vehicles. If any obstacle occurs in front of the four IR sensors the primary vehicle will be stop, if there is no obstacle the primary will movie. If the secondary vehicle transmits the emergency single, it receives the signal the primary vehicle then the vehicle automatically stops. If the secondary vehicle transmits the crash alert signal, primary vehicle receiver the signal and automatically stops. If the secondary vehicle transmits the blind spot signal, primary vehicle receives the signal and automatically stops.If it is rainy at one place, secondary vehicle passes the information to the primary vehicle. So that the vehicle can change the route.

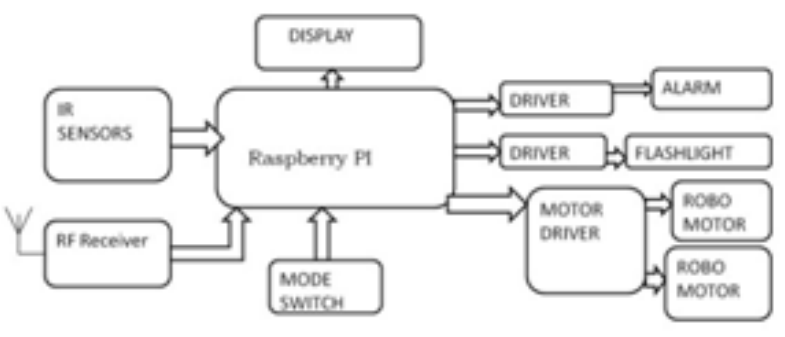

Fig1. Block scheme of Primary Vehicle

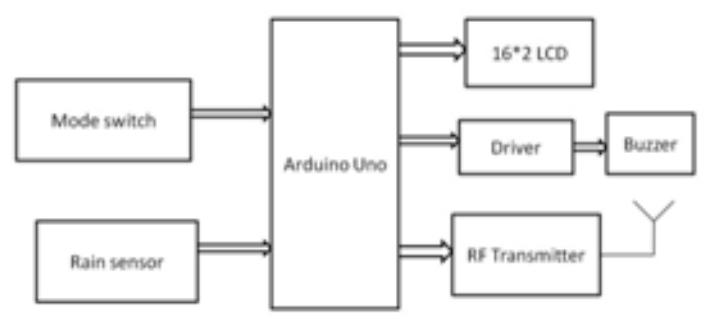

Fig2. Block scheme of Secondary Vehicle 


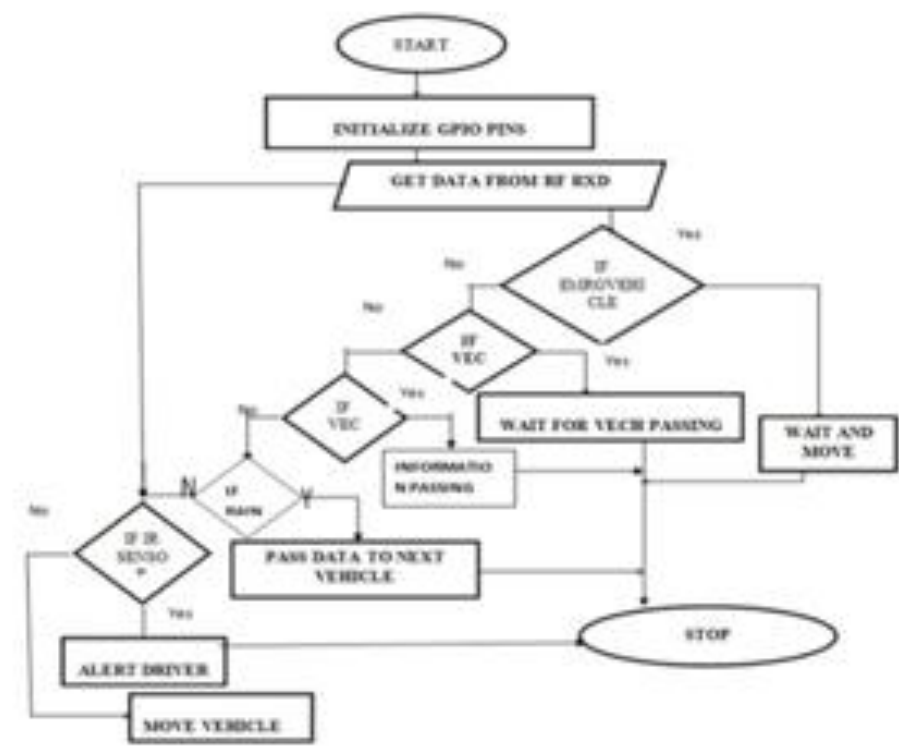

Fig3. Flow chart of the system

The above flow chart clearly explains the operation.

\subsection{Software}

In raspberry pi default language is "python". OS used in this is Jessie version. It is introduced on Jan 2017. Jessie is the name of the character in cow boy. Connect memory card to the computer to copy the software. Open win 32 disk manager. Copy Jessie image. Remove memory card and insert in raspberry pi. And update the library.

Python could be a widely used high-level programming language for general programming, created by Guido van Rossum and initial discharged in 1991. Associate degree taken language, Python encompasses a style philosophy that emphasizes code readability (notably exploitation whitespace indentation to delimit code blocks instead of premed brackets or keywords) and a syntax that permits programmers to specific ideas in fewer lines of code that may well be utilized in languages like C++ or Java. The language provides constructs supposed to alter writing clear programs on each a little and huge scale.

Python interpreter's square measure offered for several operating systems, permitting Python code to run on a large kind of systems.

In ardunio Uno default language is "embedded c". The ardunio Uno is microcontroller board based on the AT mega328 p.

\subsection{Hardware}

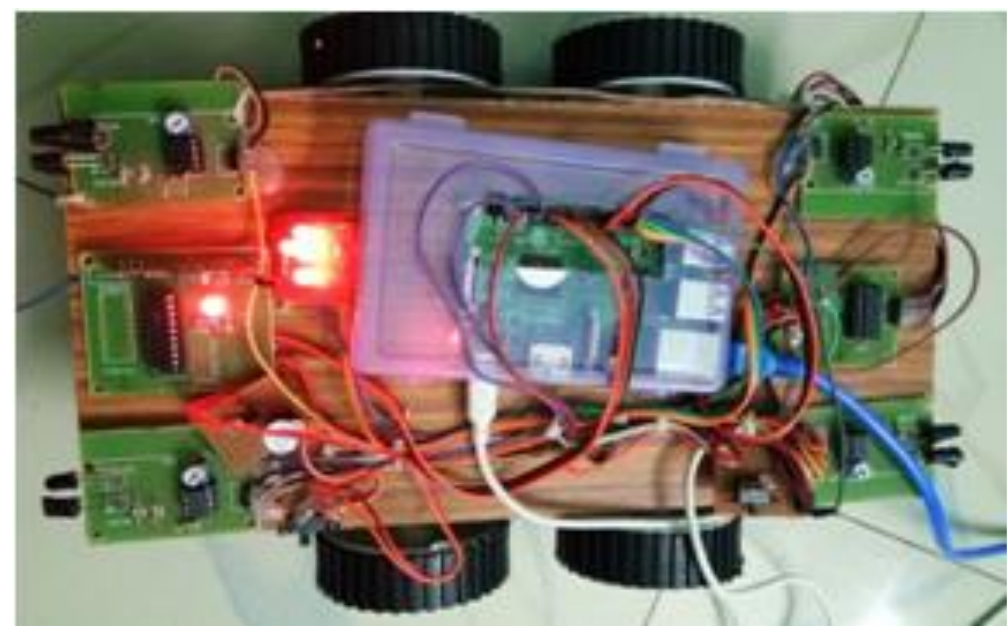

Fig4. Primary vehicle hardware 


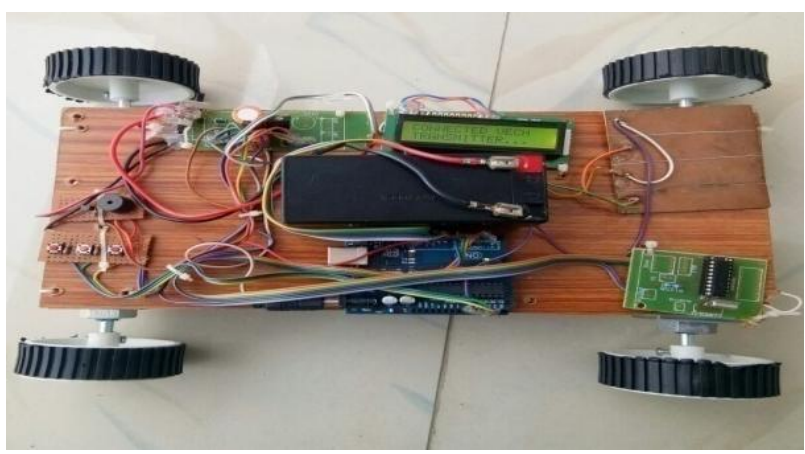

Fig5. Secondary vehicle hardware

\section{RESUlts}

In the fig no 6 If emergency vehicle which is a objective in secondary vehicle passes on the road it sends message to the primary vehicle ,the fig no 7 receiving the emergency vehicle signal from the secondary vehicle and automatically primary vehicle stops. In the fig no 8 If a vehicle coming out of the parking place, it indicates crash alert, this message can be sent to the primary vehicle ,the fig no 9 receiving the crash alert signal from the secondary vehicle and automatically primary vehicle stops .In the fig no 10 If a vehicle passes on a ' $L$ ' shaped route, it indicates blind spots that message can be sent to the primary vehicle so that the driver gets alert ,the fig 11 receiving the blind spot signal from the secondary vehicle. In the fig no 12 if it is rainy and fogy at some place that message can be sent to the primary vehicle, fig no 13 receiving the rainy and fogy at that place to change the route.fig no 14 if there is an obstacle at any side of the vehicle that message will be displayed and automatically the vehicle stops.

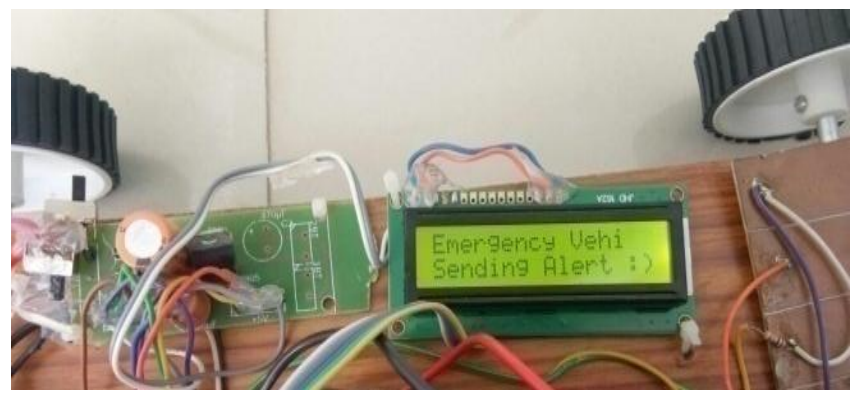

Fig6. Emergency vehicles sending

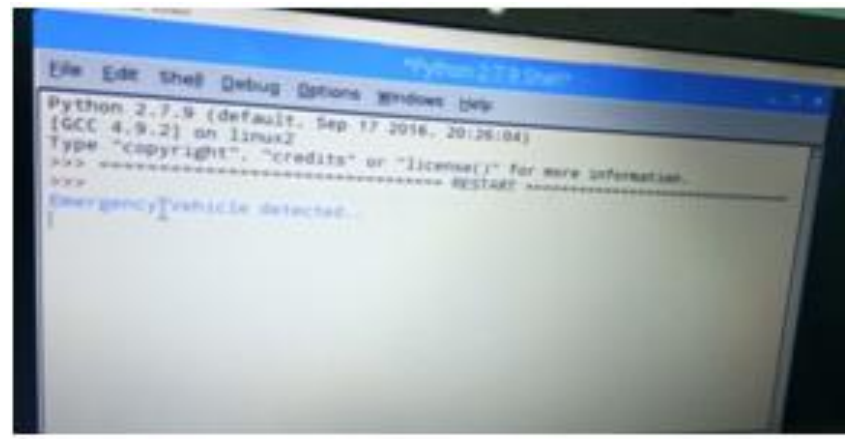

Fig7. Emergency vehicles receiving

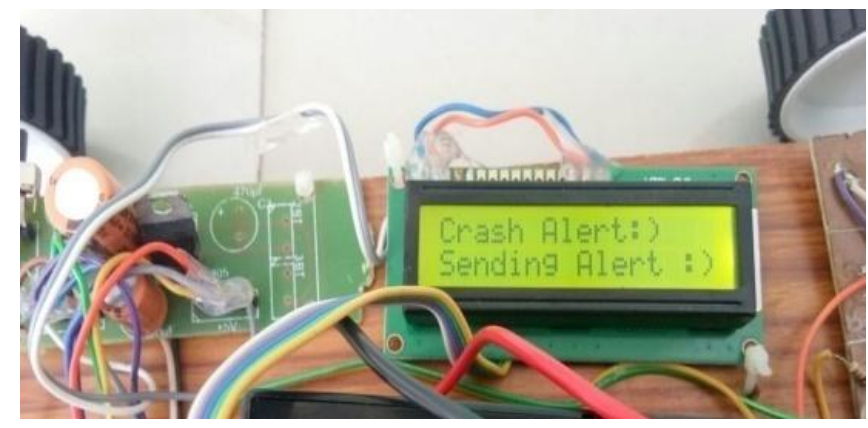

Fig8. Crash alert sending 


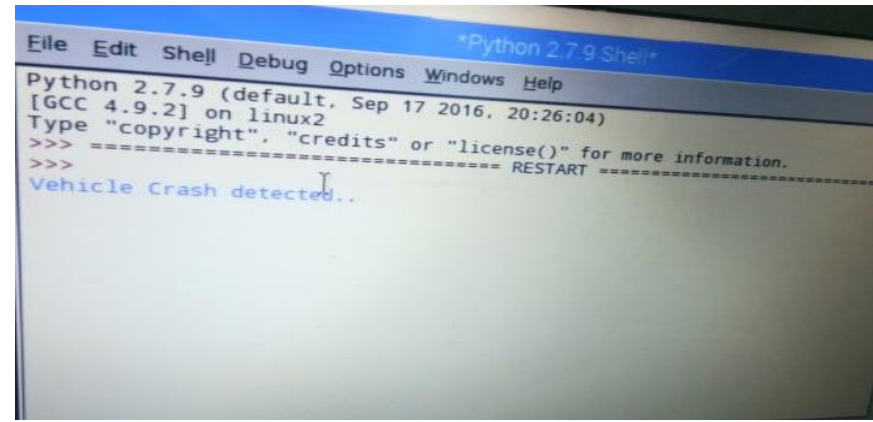

Fig9. Crash alert receiving

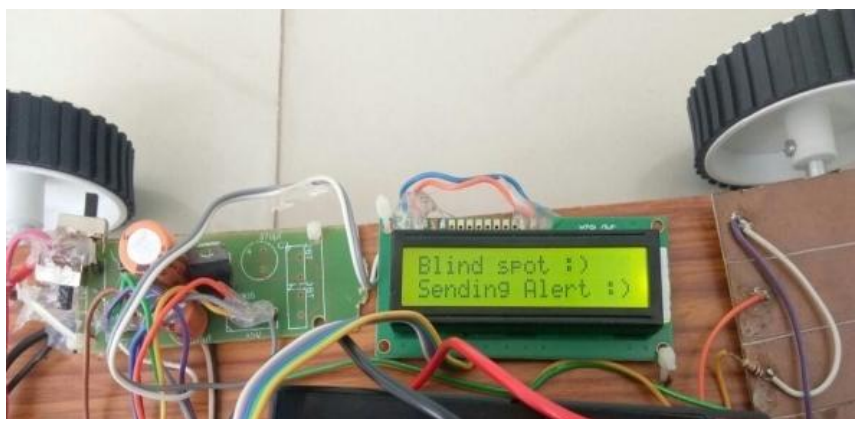

Fig10. Blind spot sending

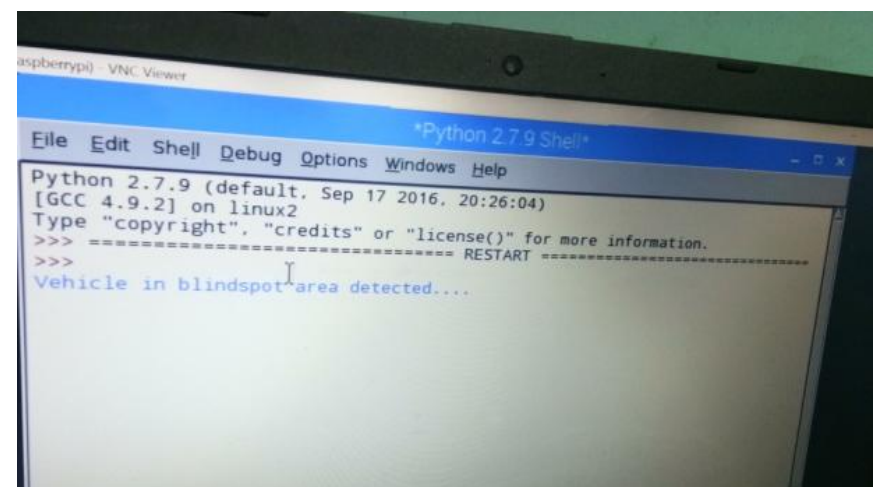

Fig11. Blind spot receiving

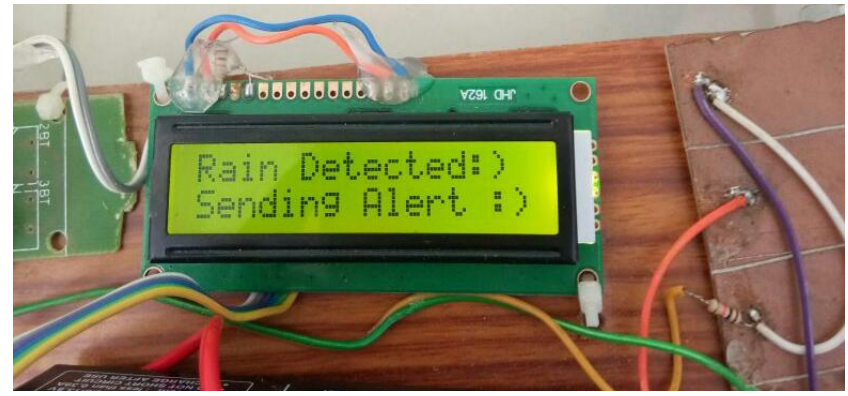

Fig12. Rain sensor sending

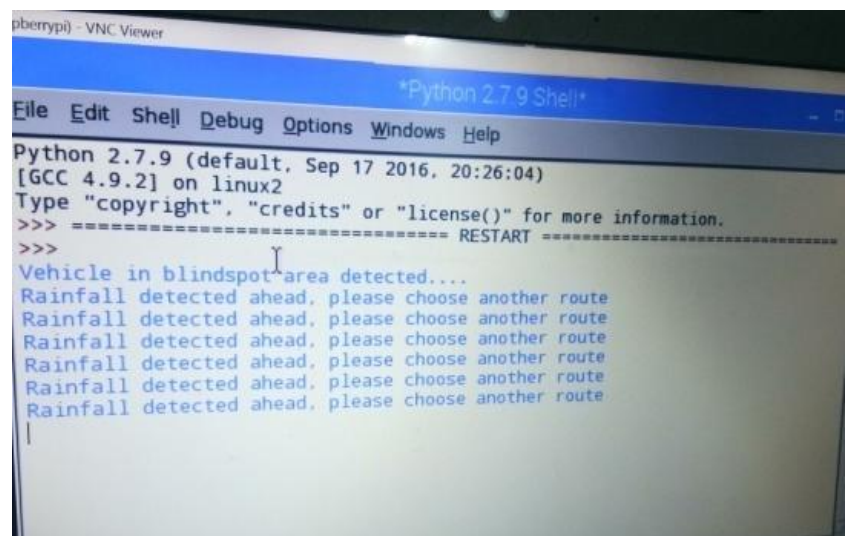

Fig13. Rain signal receiving 


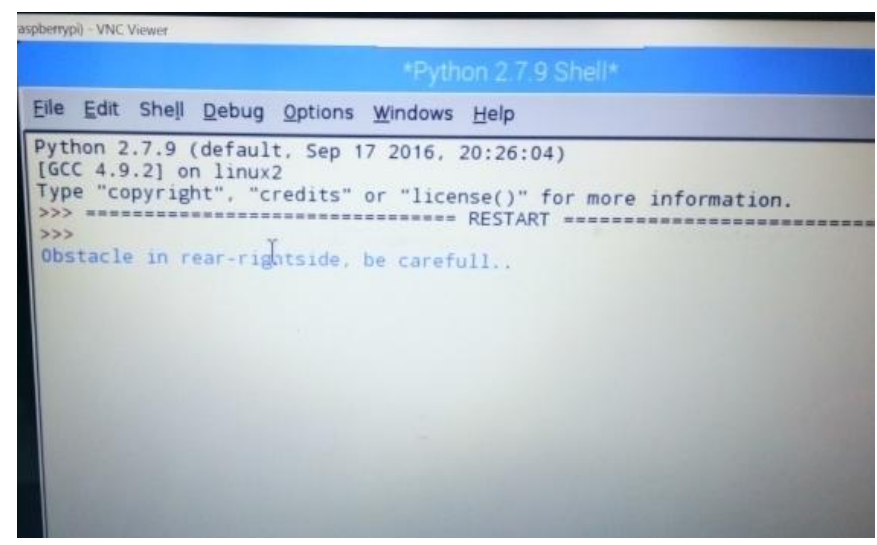

Fig14. Obstacles near

\section{CONCLUSiON}

Connected vehicle technology enables the communication between two or more vehicles. Connected vehicles will provide benefits that extend beyond drivers to include pedestrians as well as travelers using public transportation and it also promises to provide benefits in improved mobility and efficiency of our nation's transportation system. This System provides safety by avoiding crashes through notifications sent to the driver. In addition to above advantage the system also provides benefits like blind spots, crash alerts, emergency vehicle and weather monitoring.

\section{REFERENCES}

[1] Sakib Mahmud Khan Student Member, IEEE, Kakan C. Dey, Member, IEEE, and Mashrur Chowdhury, Senior Member, IEEE Real-Time Traffic State Estimation With Connected Vehicles, TRANSACTIONS ON INTELLIGENT TRANSPORTATION SYSTEMS vol.18,july 2017.

[2] Y. Ma, M. Chowdhury, M. Jeihani, and R. Fries, "Accelerated incident detection across transportation networks using vehicle kinetics and support vector machine in cooperation with infrastructure agents," IET In tell. Transp. Syst., vol. 4, no. 4, pp. 328-337, Dec. 2010.

[3] World Health Rankings, "Health Profile: Pakistan," Last accessed on 01 May 2017. [Online]. Available: http://www.worldlifeexpectancy.com/ Pakistan-road-traffic-accidents

[4] A. M. Khan and A. Tehreem, "Causes of road accidents in Pakistan," Journal of Asian Development Studies, vol. 1, no. 1, pp. 22-29, 2012.

[5] W. Yun, Z. J. Piao, G. Lei, X. Y. Wang, Y. P. Kong, and C. Jiding, "Road mortalities of vertebrate species on ring changbai mountain scenic highway, Jilin province, China." North-Western Journal of Zoology, vol. 9, no. 2, 2013.

[6] J. Kioko, C. Kiffner, P. Phillips, C. Patterson-Abrolat, W. Collinson, and S. Katers, "Driver knowledge and attitudes on animal vehicle collisions in Northern Tanzania," Tropical Conservation Science, vol. 8, no. 2, pp. 352-366, 2015.

[7] Y. Zia, M. Sabir, I. U. Saeed et al., "Pedestrian injuries and fatalities by patterns in reported road traffic crashes-Islamabad," Journal of the Pakistan Medical Association, vol. 64, no. 10, pp. 1162-1165, 2014.

[8] Gaar Law Firm, "Common types of motor vehicle accidents," Last accessed on 01 May 2017. [Online]. Available: http://www.gaarlaw.com/ Motor-Vehicle-Accidents/Car-Accidents/Car-Accident-Types.shtml

[9] Department for Transport, "Facts on pedal cy- clists," Last accessed on 01 May 2017. [On-line]. Available: https://www.gov.uk/government/uploads/system/ uploads/attachment data/file/447674/pedalcyclists-2013-data.pdf

Citation: B. Renuka et.al. (2018) "Reducing the Road Side Accidents and Collisions using the Connected Vehicle Technology", International Journal of Innovative Research in Electronics and Communications (IJIREC), 5(1), pp 24-29. DOI: http://dx.doi. org/10.20431/2349-4050.0501005

Copyright: (C) 2018 B. Renuka et al., This is an open-access article distributed under the terms of the Creative Commons Attribution License, which permits unrestricted use, distribution, and reproduction in any medium, provided the original author and source are credited. 